\title{
INTEGRAL INEQUALITIES FOR ALGEBRAIC AND TRIGONOMETRIC POLYNOMIALS
}

\author{
VITALII V. ARESTOV, POLINA YU. GLAZYRINA
}

\begin{abstract}
We investigate sharp estimates of integral functionals for operators on the set $\mathbf{T}_{n}$ of real trigonometric polynomials $f_{n}$ of degree $n \geq 1$ by the uniform norm $\left\|f_{n}\right\|_{C_{2 \pi}}$ of the polynomials and similar questions for algebraic polynomials on the unit circle of the complex plane. P. Erdös, A.P. Calderon, G. Klein, L.V. Taikov, and others investigated such inequalities. In this paper, we, in particular, show that, for $0 \leq q<\infty$, the sharp inequality $\left\|D^{\alpha} f_{n}\right\|_{L_{q}} \leq n^{\alpha}\|\cos t\|_{L_{q}}\left\|f_{n}\right\|_{\infty}$ holds on the set $\mathbf{T}_{n}$ for the Weyl fractional derivatives $D^{\alpha} f_{n}$ of order $\alpha \geq 1$. For $q=\infty(\alpha \geq 1)$, this fact was proved by P.I. Lizorkin (1965). For $1 \leq q<\infty$ and positive integer $\alpha$, the inequality was proved by Taikov (1965); however, in this case, the inequality follows from results of an earlier paper by Calderon and Klein (1951).
\end{abstract}

\section{INTRODUCTION}

Let $\mathbf{T}_{n}, n \geq 0$, be the set of trigonometric polynomials

$$
f_{n}(t)=a_{0}+\sum_{k=1}^{n}\left(a_{k} \cos k t+b_{k} \sin k t\right), \quad a_{k}, b_{k} \in \mathbb{R},
$$

of degree at most $n$ with real coefficients; for polynomial (1), we denote by $\widetilde{f}_{n}$ its conjugate polynomial

$$
\tilde{f}_{n}(t)=\sum_{k=1}^{n}\left(a_{k} \sin k t-b_{k} \cos k t\right)
$$

For $0 \leq q \leq+\infty$, we consider a functional $\|\cdot\|_{q}$ defined on the set $\mathbf{T}_{n}$ by the following relations depending on values of $q$ :

$$
\begin{gathered}
\left\|f_{n}\right\|_{q}=\left(\frac{1}{2 \pi} \int_{0}^{2 \pi}\left|f_{n}(t)\right|^{q} d t\right)^{\frac{1}{q}}, \quad 0<q<\infty, \\
\left\|f_{n}\right\|_{\infty}=\left\|f_{n}\right\|_{C_{2 \pi}}=\lim _{q \rightarrow+\infty}\left\|f_{n}\right\|_{q}=\max \left\{\left|f_{n}(t)\right|: t \in \mathbb{R}\right\}, \\
\left\|f_{n}\right\|_{0}=\lim _{q \rightarrow+0}\left\|f_{n}\right\|_{q}=\exp \left(\frac{1}{2 \pi} \int_{0}^{2 \pi} \ln \left|f_{n}(t)\right| d t\right) .
\end{gathered}
$$

Part of this work was done while the second author visited the Centre de Recerca Matemàtica, Spain. This work was also supported by the Russian Foundation for Basic Research (project no. 11-01-00462). 
Sharp inequalities for trigonometric polynomials with respect to these and more general functionals is a wide part of function theory. Below, we observe only some of them directly related to results of this paper; sufficiently complete review of this theme can be found, for example, in monographs by Rahman and Schmeisser $[18,19]$.

In 1951, Calderon and Klein [7] have obtained the following result.

Theorem A. [7] Suppose that $\varphi$ is a nonnegative function defined for nonnegative $u$ and satisfying the condition that $(\varphi(u)-\varphi(0)) / u$ be a nondecreasing function of $u, u \geq 0$. Then, the maximum of the integral

$$
\int_{0}^{2 \pi} \varphi\left(\left|f_{n}^{\prime}(t)\right|\right) d t
$$

for all $f_{n} \in \mathbf{T}_{n}$ such that $\left\|f_{n}\right\|_{\infty} \leq 1$ is achieved by the polynomial $\cos (n t+a)$, $a \in \mathbb{R}$. If, in addition, $\varphi$ is not a constant function, then $\cos (n t+a), a \in \mathbb{R}$, is the only such polynomial achieving this maximum.

The function $\varphi(u)=\sqrt{1+u^{2}}$ satisfies the assumptions of Theorem A. For this function, integral (3) is the length of the graph of the polynomial $f_{n}$ on the period $[0,2 \pi]$. In this case, the problem was posed and solved by Erdös in 1939 [8]. For convex nondecreasing functions $\varphi$, inequality (3) was rediscovered by Taikov [25]. Theorem A was rediscovered by Kristiansen [12]. Bojanov and Naidenov [6] extended Theorem A to integrals along intervals of arbitrary length.

For $q \geq 1$, the function $\varphi(u)=u^{q}, u \geq 0$, satisfies the assumptions of Theorem $\mathrm{A}$ as well as the assumptions of Theorem 1 from a later paper by Taikov [25]. Therefore, the following inequality is valid for $q \geq 1$ :

$$
\left\|f_{n}^{\prime}\right\|_{q} \leq n\|\cos n t\|_{q}\left\|f_{n}\right\|_{\infty}, \quad f_{n} \in \mathbf{T}_{n} .
$$

From this, by the classical Bernstein inequality

$$
\left\|f_{n}^{\prime}\right\|_{\infty} \leq n\left\|f_{n}\right\|_{\infty}, \quad f_{n} \in \mathbf{T}_{n}
$$

it follows that the following inequality is valid for any integer $r \geq 1$ [25]:

$$
\left\|f_{n}^{(r)}\right\|_{q} \leq n^{r}\|\cos n t\|_{q}\left\|f_{n}\right\|_{\infty}, \quad f_{n} \in \mathbf{T}_{n} .
$$

Inequality (6) and, in particular, (4) are sharp and turn into an equality only for polynomials of the form $A \cos (n t+a), A, a \in \mathbb{R}$.

The function $\varphi(u)=u^{q}$ for $0<q<1$ and, a fortiori, the function $\varphi(u)=\ln u$ do not satisfy the assumptions of Theorem A. It is proved in [22, Theorem 2] that the following different metrics inequality holds for any $q>0$ and polynomials $f_{n} \in \mathbf{T}_{n}$ all zeros of which are real:

$$
\left\|f_{n}\right\|_{q} \leq\|\cos n t\|_{q}\left\|f_{n}\right\|_{\infty} .
$$

This result and Bernstein's inequality (5) imply the validity of inequality (4) and, as a consequence, of inequality (6) in the case $0<q<1$ for polynomials $f_{n} \in \mathbf{T}_{n}$ with the property that all zeros of the derivative $f_{n}^{\prime}$ are real. In [17], it 
is proved that, for $0 \leq q<1$, inequality (4) is valid for any polynomial $f_{n} \in \mathbf{T}_{n}$ whose derivative has at most $\frac{\pi n}{2}$ zeros on the period as well as for the whole set of polynomials $\mathbf{T}_{n}$ for $1 \leq n \leq 4$.

In this paper, we prove inequality (6) for $0 \leq q<1$, all $n \geq 1$ and Weyl fractional derivatives of order $r \geq 1$; we extend this inequality to a more general class of functions $\varphi$ and a more general class of operators on the set $\mathbf{T}_{n}$.

\section{BASIC DEFINITIONS AND PRELIMINARY RESULTS}

Let $\mathbf{P}_{n}, n \geq 0$, be the set of algebraic polynomials in complex variable of degree at most $n$ with real constant term and complex other coefficients:

$$
P_{n}(z)=\sum_{k=0}^{n} c_{k} z^{k}, \quad c_{0} \in \mathbb{R}, \quad c_{k} \in \mathbb{C}, \quad 1 \leq k \leq n .
$$

We write the coefficients $c_{k}, 1 \leq k \leq n$, of polynomial (8) in the form $c_{k}=$ $a_{k}-i b_{k}$, where $a_{k}, b_{k} \in \mathbb{R}$; we also set $a_{0}=c_{0}$. The following relation is valid for polynomial (8) on the unit circle:

$$
P_{n}\left(e^{i t}\right)=f_{n}(t)+i \widetilde{f}_{n}(t),
$$

where

$$
f_{n}(t)=\operatorname{Re} P_{n}\left(e^{i t}\right), \quad \widetilde{f}_{n}(t)=\operatorname{Im} P_{n}\left(e^{i t}\right)
$$

are trigonometric polynomial (1) and its conjugate polynomial (2), respectively. It is easily understood that formulas (9)-(10) set one-to-one correspondence between the sets $\mathbf{T}_{n}$ and $\mathbf{P}_{n}$ for any $n \geq 0$.

Let $\mathfrak{B}_{n}$ be the class of nonzero (not necessarily linear) operators $L$ from the set $\mathbf{P}_{n}$ to the set $\mathbf{P}=\bigcup_{m \geq 0} \mathbf{P}_{m}$ of all algebraic polynomials with real constant term with the following properties.

1) For any polynomial $P_{n} \in \mathbf{P}_{n}$, the polynomial $L P_{n}$ has the zero constant term,

$$
L P_{n}(0)=0, \quad P_{n} \in \mathbf{P}_{n},
$$

i.e., the operator $L$ maps $\mathbf{P}_{n}$ to the set $\mathbf{P}^{0}$ of algebraic polynomials with zero constant term.

2) The operator $L$ is positive homogeneous; more precisely, the following equality holds for any polynomial $P_{n} \in \mathbf{P}_{n}$ and any nonnegative real number $\rho$ (on the unit circle at least):

$$
\left(L\left(\rho P_{n}\right)\right)(z)=\rho\left(L P_{n}\right)(z), \quad|z|=1 .
$$

3) For the operator $L$, the value

$$
\|L\|=\sup _{P_{n} \in \mathbf{P}_{n}} \frac{\left\|L P_{n}\right\|}{\left\|\operatorname{Re} P_{n}\right\|}
$$


is finite; here and below, we use the notation

$$
\|g\|=\|g\|_{\infty}=\max \{|g(z)|:|z|=1\}=\max \left\{\left|g\left(e^{i t}\right)\right|: t \in[0,2 \pi]\right\}
$$

if $g$ is an algebraic polynomial or the real part of an algebraic polynomial.

The polynomial $P_{n} \in \mathbf{P}_{n}$ is uniquely defined by the real part of its values on the unit circle, i.e., by the trigonometric polynomial $f_{n}(t)=\operatorname{Re} P_{n}\left(e^{i t}\right)$. Consequently, $L P_{n}$ is an operator of $f_{n}(t)=\operatorname{Re} P_{n}\left(e^{i t}\right)$ and value (13) can be interpreted as the "uniform norm" of this operator.

The set of operators $\mathfrak{B}_{n}$ can be described in other terms. Let $\mathbf{T}^{0}$ be the set of all real trigonometric polynomials with zero constant term. We denote by $\mathfrak{B}_{n}^{t r}$ the set of operators $L$ from $\mathbf{T}_{n}$ to $\mathbf{T}^{0}$ which are positive homogeneous (more precisely, having the property $\left.L\left(\rho f_{n}\right)=\rho L\left(f_{n}\right), f_{n} \in \mathbf{T}_{n}, \rho \geq 0\right)$ and such that the value

$$
\|L\|=\sup _{f_{n} \in \mathbf{T}_{n}} \frac{\max _{t \in \mathbb{R}} \sqrt{\left(L f_{n}(t)\right)^{2}+\left(\widetilde{L f_{n}}(t)\right)^{2}}}{\left\|f_{n}\right\|_{\infty}}
$$

is finite.

An operator $L \in \mathfrak{B}_{n}$ generates an operator from $\mathbf{T}_{n}$ to $\mathbf{T}^{0}$ (we denote this operator by the same letter $L$ ) by the formula

$$
L f_{n}(t)=\operatorname{Re}\left(L P_{n}\left(e^{i t}\right)\right), \quad f_{n} \in \mathbf{T}_{n} ;
$$

here, the polynomial $P_{n}=P_{f_{n}}$ is defined by equality (9). It is easily understood that operator (15) belongs to the set $\mathfrak{B}_{n}^{t r}$ and values (13) and (14) coincide for these operators. Conversely, the formula

$$
L P_{n}\left(e^{i t}\right)=L f_{n}(t)+i \widetilde{L f_{n}}(t), \quad f_{n}(t)=\operatorname{Re} P_{n}\left(e^{i t}\right), \quad P_{n} \in \mathbf{P}_{n},
$$

assigns to the operator $L \in \mathfrak{B}_{n}^{t r}$ an operator $L: \mathbf{P}_{n} \rightarrow \mathbf{P}^{0}$ from the class $\mathfrak{B}_{n}$; moreover, relation (15) holds. Thus, formulas (15) and (16) define a natural bijection between the sets $\mathfrak{B}_{n}$ and $\mathfrak{B}_{n}^{t r}$. Taking this fact into account, we identify the sets $\mathfrak{B}_{n}$ and $\mathfrak{B}_{n}^{\text {tr }}$ and denote them by $\mathfrak{B}_{n}$.

Let us mention that if $L: \mathbf{P}_{n} \rightarrow \mathbf{P}^{0}$ is a linear operator, then it satisfies (12) and has the finite norm (13). Thus, $L \in \mathfrak{B}_{n}$. Moreover, for operator $L$ the equality

$$
\widetilde{L f}_{n}=L \widetilde{f}_{n}, \quad f_{n} \in \mathbf{T}_{n},
$$

holds, because if $f_{n}(t)=\operatorname{Re} P_{n}\left(e^{i t}\right), P_{n} \in \mathbf{P}_{n}$, then

$$
\widetilde{L f_{n}}(t)=\operatorname{Im}\left(L P_{n}\left(e^{i t}\right)\right)=\operatorname{Re}\left(-i L P_{n}\left(e^{i t}\right)\right)=\operatorname{Re}\left(L\left(-i P_{n}\right)\right)\left(e^{i t}\right)=\left(L \widetilde{f}_{n}\right)(t) .
$$

Let $\Phi^{+}=\Phi^{+}(0,1]$ be the class of functions $\varphi$ defined on $(0,1]$ and representable in the form $\varphi(u)=\psi(\ln u)$, where the function $\psi(v)=\varphi\left(e^{v}\right)$ is continuous, nondecreasing, and convex on $(-\infty, 0]$. For example, all nondecreasing convex functions, the functions $u^{p}$ for $p>0, \ln u, \ln ^{+} u=\max \{0, \ln u\}$, and $\ln \left(1+u^{p}\right)$ for 
$p>0$ belong to the class $\Phi^{+}$. Taking into account properties of convex functions, we can assert that a function $\varphi$ defined on the half-open interval $(0,1]$ belongs to the class $\Phi^{+}(1)$ if and only if it is continuous, nondecreasing on $(0,1]$, and differentiable everywhere on $(0,1]$ except for at most countable set of points at each of which the function $\varphi$ has both one-sided derivatives; moreover, the function $u \varphi^{\prime}(u)$ is (nonnegative and) nondecreasing on $(0,1)$; more precisely, the inequality $0 \leq u_{1} \varphi_{+}^{\prime}\left(u_{1}\right) \leq u_{2} \varphi_{-}^{\prime}\left(u_{2}\right)$ holds for any $0<u_{1}<u_{2}<1$.

The class of functions $\Phi^{+}(0, \infty)$ on the half-line $(0, \infty)$ with the properties listed above was introduced in $[1,2]$, where the Bernstein inequality and its generalizations in the spaces $L_{p}$ for $p \in[0,1$ ) (and more general spaces) were studied. In [9], it is shown that it is natural to use this class in this theme.

The following assertion contains an equivalent description of the class $\Phi^{+}$.

Lemma 1. For a function $\varphi:(0,1] \rightarrow \mathbb{R}$, the following two conditions are equivalent.

(1) The function $\varphi$ is represented in the form $\varphi(u)=\psi(\ln u), u \in(0,1]$, where the function $\psi(v)=\varphi\left(e^{v}\right)$ is continuous, nondecreasing, and convex on $(-\infty, 0]$.

(2) The function $\varphi$ is (left) continuous at the point $r=1$; there exists a finite or equal to $-\infty$ right-hand limit $c=\lim _{r \rightarrow 0+} \varphi(r)$ at the point 0 and, if we set $\varphi(0)=c$, the function $\phi(z)=\varphi(|z|)$ becomes subharmonic in the unit disk $D=\{z \in \mathbb{C}:|z|<1\}$.

Proof. The lemma may be regarded known; the following reasoning easily imply it. The implication $(1) \Rightarrow(2)$. The function $\ln |z|$ is harmonic in the domain $D_{0}=\{z \in \mathbb{C}: 0<|z|<1\}$; hence (see, for example, [10, Theorem 2.2]), the composition $\psi(\ln |z|)=\varphi(|z|)=\phi(z)$ is a subharmonic function in $D_{0}$. Since the function $\varphi$ is nondecreasing on $(0,1]$, there exists a finite or equal to $-\infty$ right-hand limit $c=\lim _{r \rightarrow 0+} \varphi(r)$ at the point 0 . We set $\phi(0)=c$; then, evidently, the function $\phi$ becomes subharmonic in the unit disk $D=\{z \in \mathbb{C}:|z|<1\}$.

The implication $(2) \Rightarrow(1)$. For all $r \in(0,1)$, the following equality is valid:

$$
\varphi(r)=\sup _{|z|=r} \phi(z) \text {. }
$$

Therefore (see [10, Theorem 2.13]), $\varphi(r)$ is a convex function of $\ln r$ on any closed interval $\left[r_{1}, r_{2}\right], 0<r_{1}<r_{2}<1$. Consequently, $\varphi(r)$ is a convex function of $\ln r$ on the interval $(0,1)$ and, in view of its continuity at the point $r=1$, on the half-open interval $(0,1]$. By the same Theorem 2.13 from [10], the function $\varphi$ is nondecreasing on $[0,1)$ and, hence, on $[0,1]$.

\section{THE MAIN RESULT}

Lemma 2. For all $z \in \mathbb{C}$, the inequality

$$
|\operatorname{Re} z| \leq \frac{\left|z^{2}+1\right|}{2}
$$

is valid. It turns into an equality if and only if $|z|=1$. 
Proof. We write inequality (18) in the form

$$
\left|z^{2}+1\right|^{2}-(2 \operatorname{Re} z)^{2} \geq 0
$$

and pass to the exponential notation of complex numbers: $z=r e^{i t}$, where $r=|z|$ and $t=\arg z$. Then, we have

$$
\begin{gathered}
(2 \operatorname{Re} z)^{2}=(2 r \cos t)^{2}=4 r^{2}(\cos t)^{2}=2 r^{2}(1+\cos 2 t), \\
\left|z^{2}+1\right|^{2}=\left|r^{2} \cos 2 t+i r^{2} \sin 2 t+1\right|^{2}=r^{4}+2 r^{2} \cos 2 t+1,
\end{gathered}
$$

and, finally,

$\left|z^{2}+1\right|^{2}-(2 \operatorname{Re} z)^{2}=r^{4}+2 r^{2} \cos 2 t+1-2 r^{2}-2 r^{2} \cos 2 t=r^{4}+1-2 r^{2}=\left(r^{2}-1\right)^{2}$.

The inequality is proved. The cases of equality are obvious.

In the proof of following theorem, we use the notion of subordination of two meromorphic functions going back to Littlewood and properties of subordinated functions [10, Sect. 2.8]. For functions $f$ and $F$ meromorphic in the unit disk $D=\{z \in \mathbb{C}:|z|<1\}$, we say that the function $f$ is subordinated to the function $F$ if $f(z)=F(w(z)), z \in D$, where $w$ is an analytic function in the unit disk with the property $|w(z)| \leq|z|, z \in D$. Thus, the function $w$ must satisfy the assumptions of the Schwartz lemma (see, for example, [21, Ch. 12, Theorem 12.2], $[14$, Ch. 3, Sect. 6.2]).

Theorem 1. Let $\varphi \in \Phi^{+}$. Then, the following inequality is valid for any polynomial $P \in \mathbf{P}$ with the property $P(0)=0$ :

$$
\int_{0}^{2 \pi} \varphi\left(\frac{\left|\operatorname{Re} P\left(e^{i t}\right)\right|}{\|P\|}\right) d t \leq \int_{0}^{2 \pi} \varphi(|\cos t|) d t .
$$

For the polynomials

$$
c z^{n}, \quad c \in \mathbb{C} \backslash\{0\}, \quad n \in \mathbb{N},
$$

inequality (19) turns into an equality. If the function $\varphi$ is strictly increasing, then there are no other cases of equality.

Proof. Without loss of generality, we assume that $\|P\|=1$. Since the function $\varphi$ is not decreasing, by (18), the following inequality holds:

$$
\int_{0}^{2 \pi} \varphi\left(\left|\operatorname{Re} P\left(e^{i t}\right)\right|\right) d t \leq \int_{0}^{2 \pi} \varphi\left(\frac{\left|P^{2}\left(e^{i t}\right)+1\right|}{2}\right) d t .
$$

Moreover, if $\varphi$ is increasing (i.e., strictly increasing) and $\left|P\left(e^{i t}\right)\right| \not \equiv\|P\|=1$, then inequality (21) is strict. Only the polynomials $P(z)=c z^{n},|c|=1, n \in \mathbb{N}$, have the property $\left|P\left(e^{i t}\right)\right| \equiv 1$. Therefore, if the function $\varphi$ is increasing, then inequality (21) may turn into an equality only for polynomials (20).

The polynomial $P$ is analytic in $\mathbb{C}, P(0)=0$, and $|P(z)| \leq 1$ for $|z| \leq 1$. By the Schwartz lemma, the inequality $|P(z)| \leq|z|,|z| \leq 1$, is also valid. Therefore, 
the function $\left(P^{2}(z)+1\right) / 2$ is subordinated to the function $\left(z^{2}+1\right) / 2$ in the disk $|z|<1$. By Theorem 2.23 from [10], we have

$$
\int_{0}^{2 \pi} \varphi\left(\left|\frac{P^{2}\left(e^{i t}\right)+1}{2}\right|\right) d t \leq \int_{0}^{2 \pi} \varphi\left(\left|\frac{e^{2 i t}+1}{2}\right|\right) d t=\int_{0}^{2 \pi} \varphi(|\cos t|) d t .
$$

Relations (21) and (22) imply (19).

Evidently, inequality (19) turns into an equality for polynomials (20). The theorem is proved.

Theorem 1 allows us to justify the following assertion.

Theorem 2. Let $\varphi \in \Phi^{+}$and $L \in \mathfrak{B}_{n}$. Then, the following inequality is valid:

$$
\int_{0}^{2 \pi} \varphi\left(\frac{\left|\operatorname{Re} L P_{n}\left(e^{i t}\right)\right|}{\|L\| \mid\left\|\operatorname{Re} P_{n}\right\|}\right) d t \leq \int_{0}^{2 \pi} \varphi(|\cos t|) d t, \quad P_{n} \in \mathbf{P}_{n} .
$$

If, for an operator $L \in \mathfrak{B}_{n}$, there exists a polynomial $P_{n}^{*} \in \mathbf{P}_{n}$ extremal in problem (13) with the property $\left(L P_{n}^{*}\right)(z)=c z^{m}$, where $c \in \mathbb{C}, c \neq 0$, and $m$ is a positive integer, then the polynomial $P_{n}^{*}$ is also extremal in inequality (23). If the function $\varphi$ is increasing on $(0,1]$, then only such polynomials are extremal in (23).

Proof. Let $\varphi \in \Phi^{+}$and $L \in \mathfrak{B}_{n}$. For an arbitrary polynomial $P_{n} \in \mathbf{P}_{n}$, the polynomial $L P_{n}$ satisfies the condition $\left(L P_{n}\right)(0)=0$. Therefore, by Theorem 1 , the following inequality holds:

$$
\int_{0}^{2 \pi} \varphi\left(\frac{\left|\operatorname{Re} L P_{n}\left(e^{i t}\right)\right|}{\left\|L P_{n}\right\|}\right) d t \leq \int_{0}^{2 \pi} \varphi(|\cos t|) d t .
$$

Definition (13) gives the estimate

$$
\left\|L P_{n}\right\| \leq\|L\|\left\|\operatorname{Re} P_{n}\right\| .
$$

Since the function $\varphi$ is not decreasing, inequality (24) implies inequality (23).

In the general case, inequalities (24) and (25) can be strict. It is seen from the proof that, if both inequalities (24) and (25) turn into equalities for a polynomial $P_{n}^{*} \in \mathbf{P}_{n}$, then this polynomial is also extremal in inequality (23). If the function

$\& \varphi$ is increasing on $(0,1]$, then the inverse statement is valid. The theorem is proved.

Theorem 2 can be formulated in the following equivalent form.

Theorem 3. Let $\varphi \in \Phi^{+}$and $L \in \mathfrak{B}_{n}$. Then, the following inequality is valid:

$$
\int_{0}^{2 \pi} \varphi\left(\frac{\left|L f_{n}(t)\right|}{\|L\|\left\|f_{n}\right\|_{\infty}}\right) d t \leq \int_{0}^{2 \pi} \varphi(|\cos t|) d t, \quad f_{n} \in \mathbf{T}_{n} .
$$

If, for an operator $L \in \mathfrak{B}_{n}$, there exists a polynomial $f_{n}^{*} \in \mathbf{T}_{n}$ extremal in problem (14) with the property $\left(L f_{n}^{*}\right)(t)=A \cos (m t+a)$, where $A, a \in \mathbb{R}, A \neq 0$, and $m$ is a positive integer, then the polynomial $f_{n}^{*}$ is also extremal in inequality (26). If the function $\varphi$ is increasing on $(0,1]$, then only such polynomials can be extremal in (26). 


\section{A Result by Rogozinski for a SPeCifiC Class of Operators}

A number of important operators on the set $\mathscr{P}_{n}$ of algebraic polynomials

$$
P(z)=\sum_{k=0}^{n} c_{k} z^{k}, \quad c_{k} \in \mathbb{C}, 0 \leq k \leq n,
$$

of degree at most $n$ with complex coefficients have the form

$$
(L P)(z)=\sum_{k=0}^{n} \gamma_{k} c_{k} z^{k},
$$

where $\left\{\gamma_{k}\right\}_{k=0}^{n}$ are complex parameters; for example, $z P^{\prime}(z)$ is such operator. In the case $\gamma_{0}=0$, operator (28) belongs to the set $\mathfrak{L}_{n}$.

Operator (28) is invariant with respect to rotations of the complex plane. More precisely, operator (28) satisfies the following equality for any $\theta \in \mathbb{R}$ :

$$
\tau_{\theta}(L P)=L\left(\tau_{\theta} P\right), \quad P \in \mathscr{P}_{n},
$$

where $\tau_{\theta}, \theta \in \mathbb{R}$, is the operator of rotation defined by the formula $\tau_{\theta} P(z)=P\left(e^{i \theta} z\right)$. We have $\left(\tau_{\theta} P\right)\left(e^{i t}\right)=P\left(e^{i(t+\theta)}\right)$; therefore, $\left\|\tau_{\theta} P\right\|_{\infty}=\|P\|_{\infty}$ and $\left\|\operatorname{Re}\left(\tau_{\theta} P\right)\right\|_{\infty}=$ $\|\operatorname{Re} P\|_{\infty}$. Hence, we can easily conclude that norm (13) of operator (28) coincides with the norm of the functional

$$
(L P)(1)=\sum_{k=0}^{n} \gamma_{k} c_{k}, \quad P \in \mathscr{P}_{n} .
$$

More precisely, the following equality holds:

$$
\|L\|=\max _{P \in \mathbf{P}_{n}} \frac{|(L P)(1)|}{\|\operatorname{Re} P\|_{\infty}} .
$$

In particular, Theorem 2 begs the question of under what conditions in (31) (and, hence, in (13)) the polynomial $P_{n}^{*}(z)=z^{s}$ is extremal for some $s, 1 \leq s \leq n$. The following theorem proved by Rogozinski [20] gives necessary and sufficient $\$$ conditions for this property.

Theorem B. [20, Theorem 2] Let $1 \leq s \leq n$. In order that the inequality

$$
\left|\sum_{k=0}^{n} \gamma_{k} c_{k}\right| \leq \operatorname{Re} \gamma_{s}
$$

should hold for all $P \in \mathbf{P}_{n}$ with the property $|\operatorname{Re} P(z)| \leq 1$ for $|z| \leq 1$, it is necessary and sufficient, that the following conditions be satisfied.

1) The following inequality is valid:

$$
\operatorname{Re}\left(\gamma_{s}+2 \sum_{k=1}^{s-1} \gamma_{s-k} z^{k}+\gamma_{0} z^{s}\right) \geq 0, \quad|z| \leq 1 .
$$


2) The following relations hold:

$$
\gamma_{s+k}=\overline{\gamma_{s-k}}, \quad 0 \leq k<s \leq n-k
$$

in particular, $\gamma_{s}>0$.

3) In the case $2 s \leq n$,

$$
\gamma_{k}=0 \quad \text { if } 0 \leq k \leq p \text { and if } 2 s-p \leq k \leq n,
$$

where $2 s=n-p$. In particular, if $3 s \leq n(p \geq s)$, then all $\gamma_{k}$ must vanish and (32) is only trivially possible.

In important special case $s=n$, condition (33) and the condition $\gamma_{n}>0$ alone remain as necessary and sufficient. Also, if, in this case, the left-hand side of (33) remains positive, then $P(z)=\eta z^{n},|\eta|=1$, are the only extremal polynomials.

For $s=n$, this result was obtained earlier by Bernstein $[3,4]$ in terms of trigonometric polynomials under the additional constraint $\operatorname{Im} \gamma_{0}=0$. Similar problems about norms of operators and functionals on $\mathbf{T}_{n}$ and $\mathbf{P}_{n}$ were also studied by Szegö [24], Shapiro [23], Zavalishchin [29], Parfenenkov [16], and others.

\section{Sharp INEQUALities FOR DERIVATIVES OF FRACTIONAL ORDER}

In this section, we give an analog of Theorem A by Calderon and Klein for functions $\varphi \in \Phi^{+}$and derivatives of fractional order.

In 1928, Szegö [24] (see also [30, Vol. 2, Ch. 10, Sect. 3]) proved the following statement.

Theorem C. [24] For any $n \geq 1$ and any real $\theta$, the following inequality holds on the set $\mathbf{T}_{n}$ :

$$
\left\|f_{n}^{\prime} \cos \theta-\widetilde{f}_{n}^{\prime} \sin \theta\right\|_{\infty} \leq n\left\|f_{n}\right\|_{\infty}, \quad f_{n} \in \mathbf{T}_{n}
$$

as a consequence, the following inequality holds:

$$
\left\|\sqrt{\left(f_{n}^{\prime}\right)^{2}+\left(\widetilde{f_{n}^{\prime}}\right)^{2}}\right\|_{\infty} \leq n\left\|f_{n}\right\|_{\infty}, \quad f_{n} \in \mathbf{T}_{n} .
$$

Inequalities (36) and (37) are sharp and turn into equalities only for the polynomials

$$
A \cos (n t+a), \text { where } A, a \in \mathbb{R} .
$$

By Theorem $\mathrm{C}$ and Bernstein's inequality (5), for any $n \geq 1$, any integer $r \geq 1$, and real $\theta$, the following inequality holds:

$$
\left\|f_{n}^{(r)} \cos \theta-\widetilde{f}_{n}^{(r)} \sin \theta\right\|_{\infty} \leq n^{r}\left\|f_{n}\right\|_{\infty}, \quad f_{n} \in \mathbf{T}_{n} ;
$$

as a consequence, the following inequality holds:

$$
\left\|\sqrt{\left(f_{n}^{(r)}\right)^{2}+\left(\widetilde{f}_{n}^{(r)}\right)^{2}}\right\|_{\infty} \leq n^{r}\left\|f_{n}\right\|_{\infty}, \quad f_{n} \in \mathbf{T}_{n} .
$$


Inequalities (39) and (40) are sharp and turn into equalities only for polynomials (38). Inequalities (39) and (40) contain, in particular, the classical (sharp) Bernstein inequality

$$
\left\|f_{n}^{(r)}\right\|_{\infty} \leq n^{r}\left\|f_{n}\right\|_{\infty}, \quad f_{n} \in \mathbf{T}_{n}
$$

Let $D^{\alpha} f_{n}$ be the Weyl fractional derivative of order $\alpha \in \mathbb{R}$ of a polynomial $f_{n}$,

$$
D^{\alpha} f_{n}(t)=\sum_{k=1}^{n} k^{\alpha}\left(a_{k} \cos (k t+\alpha \pi / 2)+b_{k} \sin (k t+\alpha \pi / 2)\right) .
$$

We denote by $B_{n}^{\alpha}$ the best constant in the Bernstein inequality

$$
\left\|D^{\alpha} f_{n}\right\|_{\infty} \leq B_{n}^{\alpha}\left\|f_{n}\right\|_{\infty}, \quad f_{n} \in \mathbf{T}_{n}
$$

for fractional derivatives. Lizorkin [13] proved that, if $\alpha \geq 1$, then $B_{n}^{\alpha}=n^{\alpha}$; i.e., that an analog of inequality (41) holds for fractional derivatives of order $\alpha \geq 1$. Wilmes [28, Theorem 4, Remark 4] showed that the following estimates are valid for $0 \leq \alpha<1$ :

$$
n^{\alpha} \leq B_{n}^{\alpha} \leq 2^{1-\alpha} n^{\alpha} .
$$

Both results also can be deduced from the paper of Boas [5] 1948. (See also [27, 4.8.61].)

Kozko [11] has obtained analog of inequalities (39) and (40) for fractional derivatives (42).

Theorem D. [11] For any $n \geq 1$, an arbitrary real $\alpha \geq 1$, and any real $\theta$, the following inequality is valid on the set $\mathbf{T}_{n}$ :

$$
\max _{t \in[0,2 \pi]}\left|D^{\alpha} f_{n}(t) \cos \theta-D^{\alpha} \widetilde{f}_{n}(t) \sin \theta\right| \leq n^{\alpha}\left\|f_{n}\right\|_{\infty}, \quad f_{n} \in \mathbf{T}_{n} ;
$$

as a consequence, the following inequality holds:

$$
\left\|\sqrt{\left(D^{\alpha} f_{n}\right)^{2}+\left(D^{\alpha} \tilde{f}_{n}\right)^{2}}\right\|_{\infty} \leq n^{\alpha}\left\|f_{n}\right\|_{\infty}, \quad f_{n} \in \mathbf{T}_{n} .
$$

Ineqaulities (44) and (45) turn into equalities for polynomials (38).

We denote by $C_{n}^{\alpha}(\theta)$ and $C_{n}^{\alpha}$ the best (i.e., the least possible) constants in the following inequalities, respectively:

$$
\begin{gathered}
\max _{t \in[0,2 \pi]}\left|D^{\alpha} f_{n}(t) \cos \theta-D^{\alpha} \tilde{f}_{n}(t) \sin \theta\right| \leq C_{n}^{\alpha}(\theta)\left\|f_{n}\right\|_{\infty}, \quad f_{n} \in \mathbf{T}_{n}, \\
\left\|\sqrt{\left(D^{\alpha} f_{n}\right)^{2}+\left(D^{\alpha} \tilde{f}_{n}\right)^{2}}\right\|_{\infty} \leq C_{n}^{\alpha}\left\|f_{n}\right\|_{\infty}, \quad f_{n} \in \mathbf{T}_{n} .
\end{gathered}
$$

Inequality (43) is a special case of (46); more precisely, $B_{n}^{\alpha}=C_{n}^{\alpha}(0)$. Theorem D means that, if $\alpha \geq 1$, then $C_{n}^{\alpha}=C_{n}^{\alpha}(\theta)=n^{\alpha}$ for any $\theta \in \mathbb{R}$. 
Exact values of $C_{n}^{\alpha}(\theta)$ and $C_{n}^{\alpha}$ for $\alpha<1$ are known only in some cases. It is rather evident that, if $n=1$, then $C_{1}^{\alpha}=C_{1}^{\alpha}(\theta)=1$ for any $\alpha \in \mathbb{R}$ and $\theta \in \mathbb{R}$. For $\alpha=0$, we have $D^{0} f_{n}=f_{n}-a_{0}$; for this operator, the following sharp inequality is valid [11, Theorem 5]:

$$
\left\|D^{0} f_{n}\right\|_{\infty} \leq \frac{2 n}{n+1}\left\|f_{n}\right\|_{\infty}, \quad f_{n} \in \mathbf{T}_{n}
$$

i.e., $C_{n}^{0}(0)=\frac{2 n}{n+1}$. Taikov [26] has found the best constant $\widetilde{C}_{n}=C_{n}^{0}(\pi / 2)$ in the inequality

$$
\left\|\widetilde{f}_{n}\right\|_{\infty} \leq \widetilde{C}_{n}\left\|f_{n}\right\|_{\infty}, \quad f_{n} \in \mathbf{T}_{n}
$$

the asymptotic behavior of this value $\widetilde{C}_{n} \sim \frac{2}{\pi} \ln n$ as $n \rightarrow \infty$ was known earlier (see, for example, [30, Vol. 1, Ch. 2, § 12]). The authors do not know other cases when the values $C_{n}^{\alpha}(\theta)$ and $C_{n}^{\alpha}$ are found. It is natural to ask the question about conditions on the parameters under which the values $C_{n}^{\alpha}(\theta)$ and $C_{n}^{\alpha}$ are equal to $n^{\alpha}$. The polynomials $f_{n}(t)=\cos n t$ and $f_{n}(t)=\cos t$ show that, for any values of the parameters, the estimates $C_{n}^{\alpha} \geq C_{n}^{\alpha}(\theta) \geq \max \left\{n^{\alpha}, 1\right\}$ hold. Consequently, the fact that inequality (44) or (45) does not hold means that the constant in inequality (46) or (47), respectively, is greater than $n^{\alpha}$.

To prove Theorem D, Kozko has constructed a quadrature formula for the operator

$$
\Lambda_{\theta}^{\alpha} f_{n}(t)=D^{\alpha} f_{n}(t) \cos \theta-D^{\alpha} \widetilde{f}_{n}(t) \sin \theta, \quad f_{n} \in \mathbf{T}_{n} .
$$

This formula has the form

$$
\Lambda_{\theta}^{\alpha} f_{n}(t)=\sum_{k=0}^{2 n-1} \mu_{k}(\alpha, \theta)(-1)^{k} f_{n}\left(t_{k}+t\right), \quad t_{k}=\frac{\pi k}{n}+\frac{\alpha \pi}{2 n}+\frac{\theta}{n},
$$

and is similar to quadrature formulas by M. Riesz and Szegö (see [24], [30, Vol. 2, Ch. 10, Sect. 3]). In [11], a quantity $\alpha_{n}(\theta)$ is given in terms of the coefficients $\left\{\mu_{k}(\alpha, \theta)\right\}_{k=0}^{2 n-1}$ such that inequality (44) is valid for all $\alpha \geq \alpha_{n}(\theta)$; the quantity $\alpha_{n}(\theta)$ satisfies the condition $0<\alpha_{n}(\theta) \leq 1$. Moreover, in [11, Theorem 3], it is proved that, for any $0<\alpha<1$ and even $n=2 m$, there exists $\theta$ such that inequality (44) and, a fortiori, inequality (45) do not hold.

Mohapatra, Qazi, and Rahman [15] investigated necessary and sufficient conditions on the parameter $\alpha$ for the validity of inequality (45). To find them, they used Theorem B by Rogozinski presented above. In [15, Theorem $\left.1^{\prime}\right]$, it is proved that inequality (45) is valid if and only if $\alpha \geq \alpha_{n}^{*}$, where

$$
\alpha_{n}^{*}=\inf _{\alpha>0}\left\{\alpha: \operatorname{Re}\left(1+\sum_{k=1}^{n}(1-k / n)^{\alpha} z^{k}\right)>1 / 2 \text { for }|z|<1\right\} .
$$

By results of paper [11], the inequality $\alpha_{n}^{*} \leq 1$ holds for any $n \geq 1$ and the equality $\alpha_{2 m}^{*}=1$ holds for even $n=2 m$. Note that Theorem 4 below implies that $\alpha_{n}^{*}=1$ for any $n \geq 2$. 
We are going to prove that inequality (45) does not hold for $\alpha<1$ not only for even $n$, as already proven in [11], but for any $n \geq 2$; more precisely, $C_{n}^{\alpha}>n^{\alpha}$. The consideration above implies that it is sufficient to do this for $0<\alpha<1$.

The following assertion was proved by Kozko [11, Theorem 3] for even $n$. We justify this assertion (for any $n \geq 2$ ) with the help of other reasonings. Here, we use results and ideas from [11, 29] and from prior publications [4, 24].

Lemma 3. For $n \geq 2,0<\alpha<1$, and $\theta=-\alpha \pi / 2$, the best constant in inequality (46) satisfies the following strict inequality:

$$
C_{n}^{\alpha}(\theta)>n^{\alpha}
$$

As a consequence, for any $n \geq 2$ and $0<\alpha<1$, inequality (45) is not valid; i.e., the sharp constant in (45) is strictly greater than $n^{\alpha}$.

Proof. For the polynomial $c_{n}(t)=\cos n t$, we have

$$
\left(\Lambda_{\theta}^{\alpha} c_{n}\right)(t)=n^{\alpha}(\cos (n t+\theta) \cos (\theta)-\sin (n t+\theta) \sin (\theta))=n^{\alpha} \cos n t .
$$

Applying quadrature formula (49), we obtain the equality

$$
n^{\alpha}=\left(\Lambda_{\theta}^{\alpha} c_{n}\right)(0)=\sum_{k=0}^{2 n-1} \mu_{k}(\alpha, \theta) .
$$

Thus,

$$
C_{n}^{\alpha}(\theta) \geq n^{\alpha}=\sum_{k=0}^{2 n-1} \mu_{k}(\alpha, \theta) .
$$

By [29, Theorem 1], inequality (50) turns into an equality if and only if all nonzero coefficients $\mu_{k}=\mu_{k}(\alpha, \theta)$ have the same sign. Let us show that this condition is not satisfied for $0<\alpha<1$. More precisely, let us prove that

$$
\begin{aligned}
& \mu_{0}(\alpha, \theta)>0, \\
& \mu_{2 j}(\alpha, \theta)<0, \quad j=1, \ldots, n-1 .
\end{aligned}
$$

We use the representation of the coefficients $\mu_{k}(\alpha, \theta)$ from [11, Lemma 2.2, formulas $(2.4),(2.3)]$ : for $k=0$,

$$
\mu_{0}(\alpha, \theta)=\frac{1}{n}\left(\sum_{\ell=1}^{n-1} \ell^{\alpha}+\frac{n^{\alpha}}{2}\right) ;
$$

for $k=2 j, j=1, \ldots, n-1$,

$$
\begin{aligned}
& \mu_{2 j}(\alpha, \theta)=\frac{\nu_{2 j}(\alpha)}{4 n \sin ^{2}(j \pi / n)} \\
& \nu_{2 j}(\alpha)=-\sum_{\ell=1}^{n-1}\left((\ell+1)^{\alpha}-2 \ell^{\alpha}+(\ell-1)^{\alpha}\right) \cos \left(\ell t_{2 j}\right)+n^{\alpha}-(n-1)^{\alpha}-1 .
\end{aligned}
$$


Evidently, $\mu_{0}(\alpha, \theta)>0$. Let us verify that $\mu_{2 j}(\alpha, \theta)<0, j=1, \ldots, n-1$. To this end, it is sufficient to show that $\nu_{2 j}(\alpha)<0$. The inequality $(\ell+1)^{\alpha}-2 \ell^{\alpha}+$ $(\ell-1)^{\alpha}<0$ holds for $0<\alpha<1$ and $\ell \geq 1$; hence,

$$
\begin{aligned}
\nu_{2 j}(\alpha) & =-\sum_{\ell=1}^{n-1}\left((\ell+1)^{\alpha}-2 \ell^{\alpha}+(\ell-1)^{\alpha}\right) \cos \left(\ell t_{2 j}\right)+n^{\alpha}-(n-1)^{\alpha}-1< \\
& <-\sum_{\ell=1}^{n-1}\left((\ell+1)^{\alpha}-2 \ell^{\alpha}+(\ell-1)^{\alpha}\right)+n^{\alpha}-(n-1)^{\alpha}-1= \\
& =-\left(n^{\alpha}-(n-1)^{\alpha}-1\right)+n^{\alpha}-(n-1)^{\alpha}-1=0 .
\end{aligned}
$$

Lemma 3 is proved.

Theorem D by Kozko and Lemma 3 allow us to state the following assertion.

Theorem 4. Inequality (45) holds for $n \geq 2$ if and only if $\alpha \geq 1$.

Remark 1. Let us discuss the cases when inequalities (44) and (45) turn into equalities for integer $n \geq 1$ and real $\alpha \geq 1$. Evidently, inequalities (44) and (45) turn into equalities for polynomials (38). By Theorem $\mathrm{C}$, for $\alpha=1$ and, as a consequence, for all integer $\alpha \geq 1$, equality in (44) and (45) is attained only by polynomials (38). In [11, Lemma 2.3], it is proved that the coefficients of quadrature formula (49) are nonnegative for $\alpha \geq 1$, i.e., $\mu_{k}(\alpha, \theta) \geq 0, k=0, \ldots, 2 n-1$. Moreover, it is seen from the proof that all the coefficients are positive for $\alpha>1$. Hence, there are also no other cases of equality for (noninteger) $\alpha>1$.

By formula (16), to operator (48) on the set of trigonometric polynomials, there corresponds the operator

$$
\Lambda_{\theta}^{\alpha} P_{n}(z)=e^{\left(i \frac{\alpha \pi}{2}+i \theta\right)} \sum_{k=1}^{n} k^{\alpha} c_{k} z^{k}
$$

on the set $\mathscr{P}_{n}$ of algebraic polynomials (27). Operator (51) has form (28). In this case, we have

$$
\left|\Lambda_{\theta}^{\alpha} P_{n}\left(e^{i t}\right)\right|=\left|\sum_{k=1}^{n} k^{\alpha} c_{k} e^{i k t}\right|=\sqrt{\left(D^{\alpha} f_{n}(t)\right)^{2}+\left(D^{\alpha} \widetilde{f}_{n}(t)\right)^{2}},
$$

where, $f_{n}(t)=\operatorname{Re} P_{n}\left(e^{i t}\right), \widetilde{f}_{n}(t)=\operatorname{Im} P_{n}\left(e^{i t}\right), P_{n} \in \mathscr{P}_{n}$. By Theorem D, for $\alpha \geq 1$, norms (13) and (14) of these operators satisfy the equality $\left\|\Lambda_{\theta}^{\alpha}\right\|=n^{\alpha}$. Applying Theorem 3, we arrive at the following assertion.

Theorem 5. Let $n \geq 1, \varphi \in \Phi^{+}, \alpha \geq 1$, and $\theta \in \mathbb{R}$. Then, for all $f_{n} \in \mathbf{T}_{n}$ such that $\left\|f_{n}\right\|_{\infty} \leq 1$, the following inequality is valid:

$$
\int_{0}^{2 \pi} \varphi\left(\left|D^{\alpha} f_{n}(t) \cos \theta-D^{\alpha} \widetilde{f}_{n}(t) \sin \theta\right| n^{-\alpha}\right) d t \leq \int_{0}^{2 \pi} \varphi(|\cos t|) d t .
$$


This inequality is sharp and turns into an equality for the polynomials $\cos (n t+a)$, $a \in \mathbb{R}$. If the function $\varphi$ is increasing on $(0,1]$, then only such polynomials are extremal.

Corollary 1. For all $n \geq 1, \alpha \geq 1, \theta \in \mathbb{R}$, and $q \in[0, \infty]$, the following inequality holds:

$$
\left\|D^{\alpha} f_{n} \cos \theta-D^{\alpha} \widetilde{f}_{n} \sin \theta\right\|_{q} \leq n^{\alpha}\|\cos t\|_{q}\left\|f_{n}\right\|_{\infty}, \quad f_{n} \in \mathbf{T}_{n}
$$

in particular, the following inequalities hold:

$$
\begin{aligned}
& \left\|D^{\alpha} f_{n}\right\|_{q} \leq n^{\alpha}\|\cos t\|_{q}\left\|f_{n}\right\|_{\infty}, \quad f_{n} \in \mathbf{T}_{n}, \\
& \left\|D^{\alpha} \tilde{f}_{n}\right\|_{q} \leq n^{\alpha}\|\cos t\|_{q}\left\|f_{n}\right\|_{\infty}, \quad f_{n} \in \mathbf{T}_{n} .
\end{aligned}
$$

These three inequalities are sharp and turn into equalities only for polynomials (38).

We recall the prehistory of inequalities (53)-(55). For $1 \leq q<\infty$ and integer $\alpha \geq 1$, inequality (54) was proved by Taikov [25]; however, in fact, it was contained in earlier paper by Calderon and Klein [7]. For $q=\infty$ and real $\alpha \geq 1$, inequality (54) was proved by Lizorkin [13]. Inequality (53) and, so, inequality (55) for $q=\infty$ and fractional derivatives of order $\alpha \geq 1$ were proved by Kozko [11].

\section{REFERENCES}

[1] V.V. Arestov, S.N. Bernstein inequalities for algebraic and trigonometric polynomials, Dokl. Akad. Nauk SSSR 246(6) (1979), 1289-1292 (in Russian); translation in Soviet Math. Dokl. 20(3) (1979), 600-603.

[2] V.V. Arestov, Integral inequalities for trigonometric polynomials and their derivatives, Izv. Akad. Nauk SSSR Ser. Mat. 45(1) (1981), 3-22 (in Russian); translation in Math. USSR-Izv. 18 (1982), 1-18.

[3] S. Bernstein, Sur un théorème de M. Szegö, Prace Mat.-Fiz. 44 (1937), 9-14 (in French).

[4] S.N. Bernstein, Collected works. Vol. II. The Constructive Theory of Functions [19311953], Izdat. Akad. Nauk SSSR, Moscow, 1954 (in Russian).

[5] R.P. Boas, Quelques généralisations d'un théorème de S. Bernstein sur la dérivée d'un polynome trigonométrique. C. R. Acad. Sci. Paris 227 (1948), 618-619.

[6] B. Bojanov, N. Naidenov, An extension of the Landau-Kolmogorov inequality. Solution of a problem of Erdös, J. Anal. Math. 78 (1999), 263-280.

[7] A.P. Calderon, G. Klein, On an extremum problem concerning trigonometrical polynomials, Studia Math. 12 (1951), 166-169.

[8] P. Erdös, An extremum-problem concerning trigonometrical polynomials, Acta Litt. Sci. Szeged 9 (1939), 113-115.

[9] P.Yu. Glazyrina, Necessary conditions for metrics in integral Bernstein-type inequalities, J. Approx. Theory 162(6) (2010), 1204-1210.

[10] W.K. Hayman, P.B. Kennedy, Subharmonic Functions. Vol. I, London Mathematical Society Monographs, No. 9. Academic Press [Harcourt Brace Jovanovich, Publishers], LondonNew York, 1976. 
[11] A.I. Kozko, The exact constants in the Bernstein-Zygmund-Szegö inequalities with fracrional derivatives and the Jackson-Nikolskii inequalitiy for trigonometric polynomials, East J. Approx. 4(3) (1998), 391-416.

[12] G.K. Kristiansen, Some inequalities for algebraic and trigonometric polynomials, J. London Math. Soc. 20(2) (1979), 300-314.

[13] P.I. Lizorkin, Estimates for trigonometric integrals and Bernstein inequality for fracrional derivatives, Izv. Akad. Nauk SSSR Ser. Mat. 4(3) (1965) 109-126.

[14] A. I. Markushevich, Theory of Analytic Functions, Hindustan Pub., New Delhi, 1963.

[15] R.N. Mohapatra, A.M. Qazi, Q.I. Rahman, On fractional order derivatives of trigonometric polynomials, East J. Approx. 13(1) (2007), 105-122.

[16] A.V. Parfenenkov, Exact inequality between uniform norms of an algebraic polynomial and its real part on concentric circles in the complex plane, Tr. Inst. Mat. Mekh. UrO RAN 16(4) (2010), 254-263 (in Russian).

[17] M.A. Qazi, Q.I. Rahman, Extensions of a result of Erdös about the arc-length of a trigonometric polynomial, J. Anal. Appl. 1(2) (2003), 85-105.

[18] Q.I. Rahman, G. Schmeisser, Les Inégalités de Markoff et de Bernshtein, Les Presses de l'Universite de Montréal, 1983.

[19] Q.I. Rahman, G. Schmeisser, Analytic Theory of Polynomials, London Mathematical Society Monographs. New Series, 26, Oxford: The Clarendon Press, Oxford University Press, 2002.

[20] W.W. Rogosinski, Extremum problems for polynomials and trigonometrical polynomials, J. Lond. Math. Soc. 29(3) (1954), 259-275.

[21] W. Rudin, Real and Complex Analysis, McGraw-Hill Book Co., New York, 1987.

[22] E.B. Saff, T. Sheil-Small, Coefficient and integral mean estimates for algebraic and trigonometric polynomials with restricted zeros, J. London Math. Soc. 9 (1974), 16-22.

[23] H.S. Shapiro, On a class of extemal problems for polynomials in the unit circle, Portugaliae mathematica 20(2) (1961), 67-93.

[24] G. Szegö, Über einen Satz des Herrn Serge Bernstein, Schriften Königsberg 5 (1928), 59-70 (in German).

[25] L.V. Taikov, A generalization of an inequality of S.N. Bernstein, Trudy Mat. Inst. Steklov 78 (1965), 43-47 (in Russian).

[26] L.V. Taikov, Conjugate trigonometric polynomials, Math. Notes 48(3-4) (1990), 10441046.

[27] A. F. Timan, Theory of approximation of functions of a real variable. International Series of Monographs in Pure and Applied Mathematics, Vol. 34 A. Pergamon Press Book. The Macmillan Co., New York 1963.

[28] G. Wilmes, On Riesz-type inequalities and K-functionals related to Riesz potentials in $\mathbb{R}^{N}$, N. Numer. Funct. Anal. Optim. 1(1) (1979), 57-77.

[29] S.T. Zavalishchin, On some extremal properties of trigonometric polynomials, Trudy Mat. Inst. Steklov 78 (1965), 3-11 (in Russian).

[30] A. Zygmund, Trigonometric Series, Vols. I, II, Cambridge University Press, New York, 1959. 
Vitalit V. Arestov

Subdepartment of Mathematical Analysis

AND THEORY OF FunCTIONS

Ural Federal University,

51 Lenin AV., 620083 EkATERINBURG, Russia

AND

Institute of Mathematics and Computer Sciences

Ural Branch of the Russian Academy of Sciences

16, S. Kovalevskaja Str., 620219 Ekaterinburg, Russia

E-mail address: Vitalii.Arestov@usu.ru

Polina Yu. Glazyrina

Centre de Recerca Matemàtica

Campus de Bellaterra

08193 Bellaterra (Barcelona), Spain

AND

Subdepartment of Mathematical Analysis

AND THEORY OF FunCTIONS

URAL FEDERAL UNIVERSITY

51 Lenin AV., 620083 EkAterinburg, Russia

E-mail address: Polina.Glazyrina@usu.ru 